\title{
NMDA-induced striatal brain damage and time-dependence reliability of thionin staining in rats
}

\author{
Benoît Haelewyn $^{\text {a }}$, Philippe Alix ${ }^{b}$, E. Maubert ${ }^{c}$, Jacques H. Abraini ${ }^{a, b, *}$ \\ ${ }^{a}$ NNOXe Pharmaceuticals, 3107 Avenue des Hôtels, Suite 18C, Québec, QC G1W 4W5, Canada \\ ${ }^{\mathrm{b}}$ Centre CYCERON, UMR 6185, Université de Caen - CNRS, BP 5229, Boulevard Becquerel, 14074 Caen Cedex, France

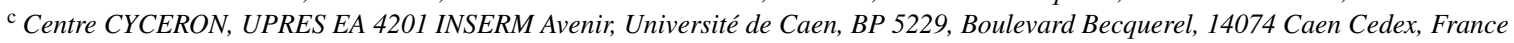

Received 31 August 2007; received in revised form 15 October 2007; accepted 24 October 2007

\begin{abstract}
Excitotoxic neuronal death induced by intracerebral injection of NMDA is a widely used model for investigating the potentially neuroprotective action of pharmacological agents against brain insults involving excitotoxic processes. Surprisingly, the time-course of NMDA-induced brain damage yet has not been investigated in the rat. Answering this question clearly needs to be assessed, given that the validity of preclinical neuroprotection studies requires to be insured that brain damage has reached a plateau that corresponds to the maximal extension of neuronal death at the time the brain is removed for histological analysis. Here, we investigated the time-course of neuronal death and the time-dependence validity of thionin coloration in rats that were given an intrastriatal injection of NMDA of $50 \mathrm{nmol}$ or 70 nmol. Our results show that, whatever the dose used, NMDA-induced brain damage reaches its maximal value $24-48 \mathrm{~h}$ after the insult. They further indicate that the volume values of brain damage as estimated by thionin coloration constitute reliable data when the brain is removed up to $48 \mathrm{~h}$ after injection of NMDA. However, if the brain is removed more than $48 \mathrm{~h}$ after the excitotoxic insult onset, there is no alternative of using other techniques, such as immunochemical or neuroimaging techniques.
\end{abstract}

(C) 2007 Elsevier B.V. All rights reserved.

Keywords: NMDA-induced neurotoxicity; Time-course in vivo; Thionin; NeuN immunochemistry

Glutamate excitotoxicity is known to play a prominent role in a variety of acute and chronic neurological injuries (Osuga and Hakim, 1994; Parsons et al., 1998). This is thought to depend largely upon the overstimulation of the $N$-methyl-D-aspartate (NMDA) and non-NMDA glutamatergic receptors (Olney, 1990; Beal et al., 1991; Cosi et al., 2004). Because of this, excitotoxic neuronal death induced by intracerebral injection of NMDA is a widely used model for investigating the potentially neuroprotective action of multiple pharmacological agents in the purpose of finding targets for neuroprotective strategies against brain insults that involved excitotoxic processes. However, surprisingly and in contrast with the time-course of transient or permanent cerebral ischemia, the time-course of NMDA-induced brain damage yet has not been investigated in the rat. Answering this ques-

\footnotetext{
* Corresponding author at: Centre CYCERON, UMR 6185, Université de Caen - CNRS, BP 5229, Boulevard Becquerel, 14074 Caen Cedex, France. Tel.: +33231470 102; fax: +33231470102.

E-mail address: abraini@cyceron.fr (J.H. Abraini).
}

tion is not trivial and clearly needs to be assessed, given that the validity and the clinical pertinence of preclinical neuroprotection studies using NMDA injection require to be insured that brain damage has reached a plateau that corresponds to the maximal extension of neuronal death at the time the brain is removed to perform histological analysis. However, following neuronal degeneration, glial cells progressively invade the brain area subjected to injury (Acarin et al., 1996), a process that can lead to "false" thionin or cresyl violet coloration (which are among the most widely used staining techniques) and thereby to false results and data interpretation. Here, we have investigated in the rat the time-course of neuronal death induced by NMDA at $50 \mathrm{nmol}$ (a dose often used in studies focusing on the potentiating effects of various agents or conditions on NMDA-induced neuronal death) and $70 \mathrm{nmol}$ (a dose often used in studies focusing on the inhibiting effects of various agents or conditions on NMDA-induced neuronal death), and further determined the time-dependence reliability of thionin coloration. Experiments were performed in eight groups of rats ( $n=7-8$ per group) that were given an intrastriatal injection of NMDA at $50 \mathrm{nmol}$ or 

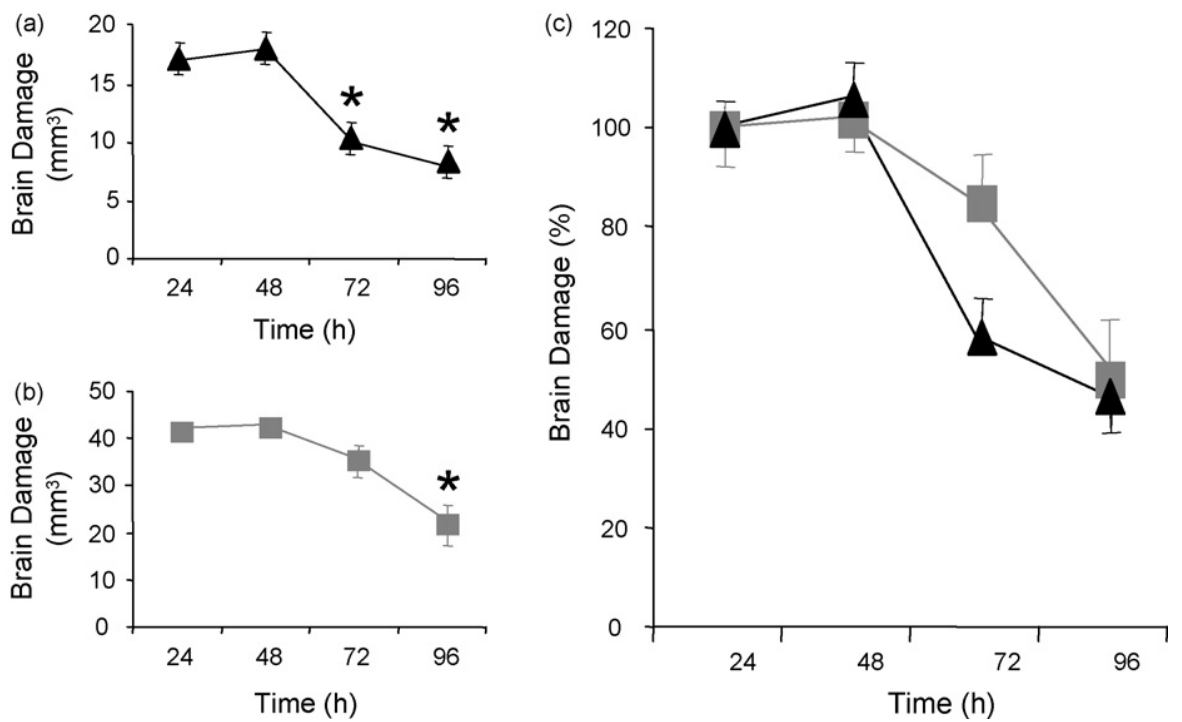

Fig. 1. Time-course of neuronal death induced by an intrastriatal injection of NMDA as assessed by thionin coloration. (A) Neuronal death induced by $50 \mathrm{nmol}$ NMDA. (B) Neuronal death induced by $70 \mathrm{nmol}$ NMDA. Whatever the dose of NMDA used, mean results show that NMDA-induced neuronal death reaches its maximal value $24 \mathrm{~h}$ after NMDA injection. Then after, the estimated volume of NMDA-induced neuronal death decreased significantly. These data indicate that volume values of brain damage obtained by thionin coloration constitute reliable data if the brain is removed up to $48 \mathrm{~h}$ after NMDA injection. (C) Comparison of the time-course of neuronal death induced by $50 \mathrm{nmol}$ and $70 \mathrm{nmol} \mathrm{NMDA}$, as expressed for each group in a percentage change of the volume value measured at $24 \mathrm{~h} . * P<0.005$ vs. neuronal death at $24 \mathrm{~h}$.

$70 \mathrm{nmol}$; then after, the volume of lesion was assessed, for each animal, at days 1-4 post-NMDA injection.

All animal-use procedures were in accordance with The Declaration of Helsinki, and were within the framework of the French legislation for the use of animals in biomedical experimentation. Adult male Sprague-Dawley rats $(n=64$; Janvier, Le Genest Saint-Isle, France) weighing 250-280 g were used. Before being used, rats were housed socially by group of four animals at $21 \pm 0.5^{\circ} \mathrm{C}$ in Perspex home cages with free access to food and water. Light was maintained on a light:dark reverse cycle, with lights on from 8:00 p.m. to 8:00 a.m. After surgery and intrastriatal injection of NMDA, the animals were housed individually in similar conditions.

On the day of surgery, rats were anesthetized with halothane (4\%) in oxygen alone in an anesthesia box, and mounted on a stereotaxic apparatus with the incisor bar set at $3.9 \mathrm{~mm}$ below the horizontal zero. Then, halothane, completed by oxygen alone, was reduced at $1.5 \%$. A burr hole was drilled and a micropipette ( $\sim 10 \mu \mathrm{m}$ at the tip) was lowered into the right striatum [according to the rat brain stereotaxic atlas of Watson and Paxinos (1998); A: $0.6 \mathrm{~mm}$; L: $3.0 \mathrm{~mm}$; V: $5.8 \mathrm{~mm}$, from bregma] in order to allow injection of $50 \mathrm{nmol}$ or $70 \mathrm{nmol} \mathrm{NMDA}$ in $1 \mu \mathrm{L} \mathrm{PBS}(\mathrm{pH}$ 7.4) over a 2 min period. After an additional 5 min period, the micropipette was removed, and the wounds were then sutured. During surgery, body temperature was kept at $37.5 \pm 0.5^{\circ} \mathrm{C}$ with a feedback controlled thermostatic heating pad (Harvard Apparatus Limited, Edenbridge, England). The animals waked up in their home cage after about $10 \mathrm{~min}$, where they had free access to food and water.

Rats were killed $24 \mathrm{~h}, 48 \mathrm{~h}, 72 \mathrm{~h}$, and $96 \mathrm{~h}$ after injection of $50 \mathrm{nmol}$ or $70 \mathrm{nmol}$ NMDA. Rats were deeply anesthetized with halothane and perfused transcardially with $50 \mathrm{~mL}$ heparinized saline solution (50 UI) followed by $150 \mathrm{~mL}$ paraformaldehyde
(4\%) in 0.01 M PBS (pH 7.4). The brain was rapidly removed, postfixed by immersion in the same fixative solution at $4{ }^{\circ} \mathrm{C}$ for $48 \mathrm{~h}$, and cryoprotected in a solution of glycerol (20\%), ethylene glycol (30\%), and PBS (50\%) for $48 \mathrm{~h}$. Then, the brain was frozen in isopentane and placed at $-80{ }^{\circ} \mathrm{C}$. Coronal brain slices $(20 \mu \mathrm{m})$ were cryostat-cut and mounted on slides. The volume of NMDA-induced neuronal death was determined by using thionin coloration as follows: slices were briefly immersed in water, stained with thionin, dehydrated with serial alcohol and cleared with xylene, and coverslipped with Eukitt ${ }^{\circledR}$ mounting. The lesioned areas were delineated by the pallor of staining in the necrotic tissue compared to the surrounding healthy tissue. In addition, a NeuN immunochemical staining was performed for comparison in rats injected with $70 \mathrm{nmol} \mathrm{NMDA}$ using the adjacent slices used for thionin. NeuN immunochemical staining was performed as follows: brain sections were washed in PBS, permeabilized with $0.1 \%$ Tween-PBS and treated with $3 \%$ bovine serum albumin (BSA)-0.1\% Tween-PBS for $45 \mathrm{~min}$, and incubated at $4{ }^{\circ} \mathrm{C}$ overnight with the primary antibody (mouse anti NeuN ref. MAB 377 1:1000 dilution, Chemicon, Paris, France) in $1 \%$ BSA-PBS. Brain slices were then incubated $1.5 \mathrm{~h}$ with a Rhodamine (TRITC) conjugated $\mathrm{F}\left(\mathrm{ab}^{\prime}\right)_{2}$ fragment donkey anti mouse IgG (ref. 715-026-150, Jackson Immunoresearch Laboratories, Interchim, Montluçon, France). Between each step, all immunosections were washed with PBS. In order to assess nonspecific binding, sections were treated identically except that the incubation with the first antibody was omitted. Both thionin slices and NeuN slices were digitized on a PC computer, and analyzed with an image analyzer (Image ${ }^{\circledR}$ Software, Scion Corp., USA). Volume of NMDA-induced brain damage was calculated by integration of the lesioned surfaces over the whole brain. Data are given as mean \pm S.E.M., and were analyzed using nonparametric statistics. Between-group comparison of data was 
made using the Kruskall-Wallis non-parametric ANOVA. Following a significant $H$ value, post hoc comparisons were further performed using the Mann-Whitney $U$-test.

An absence of thionin coloration was observed in all animals that were given an intrastriatal injection of $50 \mathrm{nmol}$ NMDA. Significant differences were found in the volume of brain damage induced by NMDA between the different groups of rats, indicating that the estimated volume of brain damage varies as a function of time $(H=20.515 ; P<0.0001)$. Estimation of brain damage by thionin was $17 \pm 1 \mathrm{~mm}^{3}$ when assessed $24 \mathrm{~h}$ after injection of NMDA, and $18 \pm 1 \mathrm{~mm}^{3}, 10 \pm 1 \mathrm{~mm}^{3}$, $8 \pm 1 \mathrm{~mm}^{3}$ when assessed respectively $48 \mathrm{~h}, 72 \mathrm{~h}$ and $96 \mathrm{~h}$ after NMDA injection (Fig. 1A). This resulted in significant differences between the estimated volume of brain damage at $24 \mathrm{~h}$ and that measured at $96 \mathrm{~h}(48 \mathrm{~h}: U=21, \mathrm{n} . s . ; 72 \mathrm{~h}$ : $U=4, P<0.01 ; 96 \mathrm{~h}: U=3.5, P<0.005)$. Similar results were obtained in rats injected with $70 \mathrm{nmol}$ NMDA. Significant differences were found in the volume of brain damage induced by NMDA between the different groups of rats, indicating that the estimated volume of brain damage varies as a function of time $(H=15.825 ; P<0.002)$. Estimation of brain damage by thionin was $42 \pm 2 \mathrm{~mm}^{3}$ when assessed $24 \mathrm{~h}$ after injection of NMDA, and $43 \pm 1 \mathrm{~mm}^{3}, 36 \pm 3 \mathrm{~mm}^{3}, 21 \pm 4 \mathrm{~mm}^{3}$ when assessed respectively $48 \mathrm{~h}, 72 \mathrm{~h}$ and $96 \mathrm{~h}$ after NMDA injection (Fig. 1B). This resulted in significant differences between the estimated volume of brain damage at $24 \mathrm{~h}$ and that measured at 96 h ( 48 h: $U=25$, n.s.; 72 h: $U=17, P<0.1 ; 96$ h: $U=3$, $P<0.005)$.

For comparison, the volume of neuronal death induced by $70 \mathrm{nmol}$ NMDA was further assessed using NeuN immunochemical staining (Fig. 2A-D). In contrast with that found with thionin, no significant difference in the volume of brain damage as assessed by NeuN staining was observed between the different groups of rats $(H=1.792$, n.s.). Estimation of brain damage was $44 \pm 2 \mathrm{~mm}^{3}$ when assessed $24 \mathrm{~h}$ after injection of NMDA, and $47 \pm 3 \mathrm{~mm}^{3}, 44 \pm 3 \mathrm{~mm}^{3}, 41 \pm 3 \mathrm{~mm}^{3}$ when assessed respectively $48 \mathrm{~h}, 72 \mathrm{~h}$ and $96 \mathrm{~h}$ after NMDA injection (Fig. 2E). As illustrated in Fig. 2E, this resulted in a significant difference between the volume values of brain damage as measured by thionin and NeuN staining $(H=23.938, P<0.002)$, thereby leading to significant differences between the estimated volume of brain damage at $72 \mathrm{~h}$ and $96 \mathrm{~h}$ post-NMDA injection $(24 \mathrm{~h}$ : $U=24$, n.s.; 48 h: $U=20$, n.s.; 72 h: $U=17, P<0.02 ; 96$ h: $U=6$; $P<0.01$ ).

Our findings indicate that whatever the dose used, either $50 \mathrm{nmol}$ or $70 \mathrm{nmol}$ NMDA, brain damage following an intrastriatal injection of NMDA reaches its maximal volume value $24-48 \mathrm{~h}$ after the insult. Then after, when assessed by thionin, the estimated volume of brain damage rapidly decreased. This is in excellent agreement with a recent investigation that has reported in mice that the estimated volume of brain damage, as measured by the cresyl violet staining technique, increased as a function of time up to $24 \mathrm{~h}$ after injection of kainic acid, remained unchanged up to $48 \mathrm{~h}$ after this excitotoxic insult, and then showed a dramatic reduction when measured $168 \mathrm{~h}$ after the insult (Cosi et al., 2004). Interestingly, similar findings have been also reported in rats subjected to cerebral ischemia by using magnetic resonance imaging and triphenyltetrazolium chloride staining techniques (Henninger et al., 2006). Taken together these data provide evidence that brain damage induced by insults that comprise an excitotoxic component shows a progressive development that reaches its maximal volume value $24-48 \mathrm{~h}$ after the insult in adult rodents. This conclusion obtained from several studies, using different staining or imaging techniques to assess neuronal death in different types of rodents, has to be emphasized since the evolving processes involved in excitotoxic neuronal death are sometimes believed to take more than $24 \mathrm{~h}$ in adult rodents. The sensitivity of pups to NMDA-induced neuronal death is well known to differ from that of adult rats. However, whether such a difference in sensitivity may influence the time-course of NMDA-induced neuronal death is unlikely to be true since our results clearly indicate that the time-course of neuronal death induced by $50 \mathrm{nmol}$ and $70 \mathrm{nmol}$ (conditions that may mimic sensitivity) is similar. In addition, our findings together with those reported by Cosi et al. (2004) further indicate that the volumes of brain damage obtained with the thionin and cresyl violet staining techniques constitute reliable data when the brain is removed up to $48 \mathrm{~h}$ after the excitotoxic insult onset.

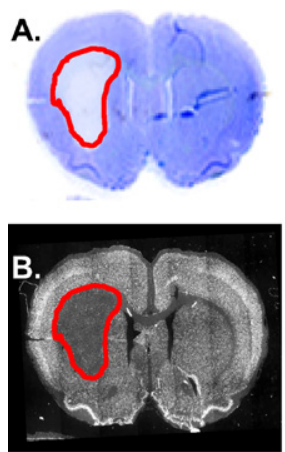

24h
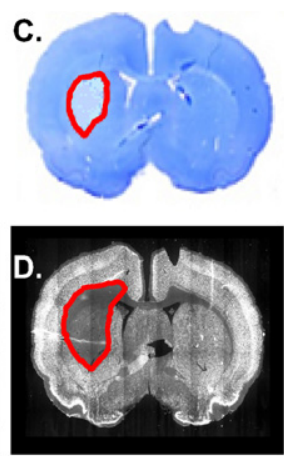

$96 \mathrm{~h}$

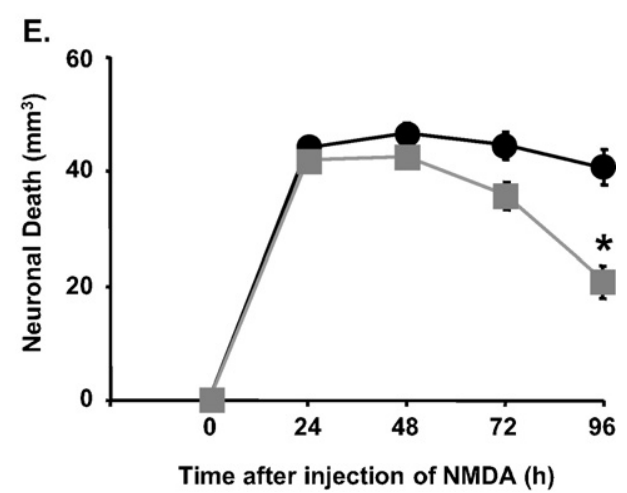

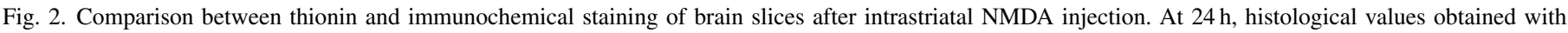

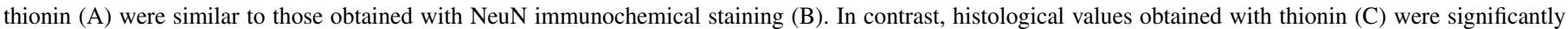

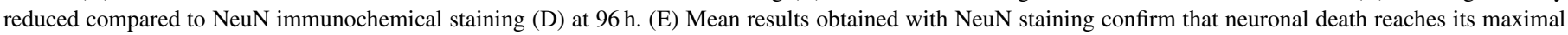

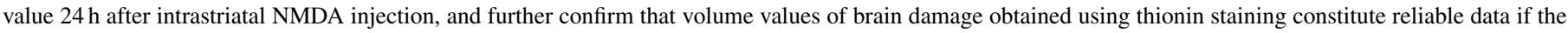
brain is removed up to $48 \mathrm{~h}$ after NMDA injection. ${ }^{*} P<0.01$ between thionin staining and NeuN staining. 
Alternatively, if the brain is removed more than $48 \mathrm{~h}$ after induction of the excitotoxic insult, thionin and cresyl violet staining techniques cannot be used confidently as indicated by the differences in volume of neuronal death measured between NeuN- and thionin staining in the present study. Doubtlessly, this can be attributed to the fact that, following brain damage, glial cells progressively invade the brain area subjected to injury (Acarin et al., 1996). Therefore, because thionin and cresyl violet unspecifically tag all cell nuclei, in contrast with NeuN staining that specifically tags the neuronal nucleus, the use of thionin and cresyl violet staining techniques will result in a false estimation of neuronal death. With no doubt, this technical bias should be taken into account in neuroprotection studies that needs to evaluate global brain histologic outcome. Therefore, while thionin and cresyl violet can be used confidently to assess neuronal death up to $48 \mathrm{~h}$ after induction of an excitotoxic insult, there is no alternative of using other techniques, such as immunochemical or neuroimaging techniques, for longer periods.

\section{References}

Acarin L, Gonzales B, Castellano B, Castro AJ. Microglial response to $N$-methylD-aspartate-mediated excitotoxicity in the immature rat brain. J Comp Neurol 1996;367:361-74.

Beal MF, Ferrante RJ, Swartz KJ, Kowall NW. Chronic quinolinic acid lesions in rats closely resemble Huntington's disease. J Neurosci 1991;11:1649_ 59.

Cosi C, Guerin K, Marien M, Koek W, Rollet K. The PARP inhibitor benzamide protects against kainate and NMDA but not AMPA lesioning of the mouse striatum in vivo. Brain Res 2004;996:1-8.

Henninger N, Kuppers-Tiedt L, Sicard KM, Gunther A, Schneider D, Schwab S. Neuroprotective effect of hyperbaric oxygen therapy monitored by MRimaging after embolic stroke in rats. Exp Neurol 2006;201:316-23.

Olney JW. Excitotoxin-mediated neuron death in youth and old age. Prog Brain Res 1990;86:37-51.

Osuga H, Hakim AM. Relevance of interstitial glutamate to selective vulnerability in focal cerebral ischemia. J Cereb Blood Flow Metab 1994;14:343-7.

Parsons CG, Danysz W, Quack G. Glutamate in CNS disorders as a target for drug development: an update. Drug News Perspect 1998;11:523-69.

Watson C, Paxinos G. The rat brain in stereotaxic coordinates. San Diego: Academic Press; 1998. 